\title{
THE JOINT DENSITY OF THE SURPLUS BEFORE AND AFTER RUIN IN THE SPARRE ANDERSEN MODEL
}

\author{
SUSAN M. PITTS, ${ }^{*}$ University of Cambridge \\ KONSTADINOS POLITIS, ${ }^{* *}$ University of Piraeus
}

\begin{abstract}
Gerber and Shiu (1997) have studied the joint density of the time of ruin, the surplus immediately before ruin, and the deficit at ruin in the classical model of collective risk theory. More recently, their results have been generalised for risk models where the interarrival density for claims is nonexponential, but belongs to the Erlang family. Here we obtain generalisations of the Gerber-Shiu (1997) results that are valid in a general Sparre Andersen model, i.e. for any interclaim density. In particular, we obtain a generalisation of the key formula in that paper. Our results are made more concrete for the case where the distribution between claim arrivals is phase-type or the integrated tail distribution associated with the claim size distribution belongs to the class of subexponential distributions. Furthermore, we obtain conditions for finiteness of the joint moments of the surplus before ruin and the deficit at ruin in the Sparre Andersen model.
\end{abstract}

Keywords: Sparre Andersen model; surplus prior to ruin; deficit at ruin; ladder height; Wiener-Hopf factors; phase-type distribution; subexponential distribution

2000 Mathematics Subject Classification: Primary 60K10; 91B30

Secondary 60K05

\section{Introduction}

For the classical model of actuarial risk theory, Dufresne and Gerber (1988) studied the joint density function of the surplus immediately prior to ruin and the deficit at ruin. More recently, Gerber and Shiu (1997) added a third quantity, namely the time of ruin, and studied the joint distribution of these three random variables. Their results have motivated extensive research on this topic; one avenue for generalising the results of that paper that has turned out to be popular in recent years is to relax the exponential assumption for the distribution of the interarrival times in the model. Thus, we are led to consider the more general Sparre Andersen model of risk theory and the obvious next step from the exponential assumption is to allow the distribution, $K$, of interclaim times to belong to the Erlang (or generalised Erlang) family of distributions. For recent results in this area under that, more general, assumption, we refer the reader to Dickson and Hipp (2000), (2001), Cheng and Tang (2003), and the accompanying discussion in Gerber and Shiu (2003a), (2003b), Li and Garrido (2004), (2005), and Gerber and Shiu (2005). The main purpose of the present paper is to obtain a formula for the density of

Received 6 January 2006; revision received 11 June 2007.

* Postal address: Statistical Laboratory, University of Cambridge, Wilberforce Road, Cambridge CB3 OWB, UK. Email address: s.pitts@statslab.cam.ac.uk

** Postal address: Department of Statistics and Insurance Science, University of Piraeus, 80 Karaoli \& Dimitriou St., Piraeus 18534, Greece. 
the surplus prior to ruin and the deficit at ruin that is valid for any distribution $K$ in the Sparre Andersen model.

More precisely, our model of consideration is defined (in usual terms) as follows. Let $X_{1}, X_{2}, \ldots$ be the sizes of the claims arriving at an insurer. These claims are assumed to be independent and identically distributed (i.i.d.) with a distribution $P$ on $(0, \infty)$. Also, let $T_{1}, T_{2}, \ldots$ be the times between successive claim arrivals, where we assume that the $T_{i}$ are again i.i.d. with distribution $K$ and independent of the $X_{i}$. We assume that the distributions $K$ and $P$ have densities $k$ and $p$, respectively, such that $k$ is bounded on finite intervals.

The insurer's surplus at time $t$ is then given by

$$
U(t)=u+c t-\sum_{i=1}^{N_{t}} X_{i},
$$

where $u$ is the initial surplus, $c$ is the premium income rate, $N_{t}$ is the number of claims that have arrived up to time $t$, so that, under the above assumptions $\left\{N_{t}: t \geq 0\right\}$ constitutes a renewal process, and the summation above is defined to be 0 if $N_{t}=0$. The time of ruin, $T$, is accordingly defined by

$$
T=\inf \{t: U(t)<0\},
$$

while the surplus prior to ruin and the deficit at ruin are $U(T-)$ and, respectively, $|U(T)|$; here, the infimum of an empty set is taken to be infinite. With this convention, the probability of ruin is then defined by

$$
\psi(u)=\mathrm{P}(T<\infty \mid U(0)=u) .
$$

We assume throughout that $\mathrm{E}\left(X_{1}\right)<c \mathrm{E}\left(T_{1}\right)$, so that ruin is not certain to occur.

Now let $f(x, y, z)$ denote the joint density of $U(T-),|U(T)|$, and $T$. For a given $\delta>0$, Gerber and Shiu (1997) introduced the following functions:

$$
f(x, y \mid u)=\int_{0}^{\infty} \mathrm{e}^{-\delta t} f(x, y, t \mid u) \mathrm{d} t
$$

and

$$
f(x \mid u)=\int_{0}^{\infty} f(x, y \mid u) \mathrm{d} y .
$$

Then, for $\delta=0, f(x, y \mid u)$ is simply the joint density between $U(T-)$ and $|U(T)|$, and $f(x \mid u)$ is the marginal density of the surplus prior to ruin.

For the classical risk model, the 'key formula' in Gerber and Shiu (1997) is

$$
f(x, y \mid 0)=\lambda c^{-1} p(x+y) \mathrm{e}^{-\rho x}, \quad x>0,
$$

where $\lambda$ is the Poisson rate for arrivals and $\rho$ is the nonnegative root of the 'generalised Lundberg equation',

$$
c \rho+\lambda[\hat{p}(\rho)-1]=\delta,
$$

where $\hat{p}$ here is the Laplace transform of the function $p$. For $\delta=0$, we see that $\rho=0$, and the key formula becomes

$$
f(x, y \mid 0)=\lambda c^{-1} p(x+y), \quad x, y>0 .
$$

Large deviation results for the joint distribution of $U(T-)$ and $|U(T)|$ have been obtained by Asmussen and Klüppelberg (1996). Here, one of the main aims of this paper is to obtain an 
exact formula which parallels (2) or the more general formula (17), below, and is valid for the Sparre Andersen model. Our main results give an expression for $f(x, y \mid 0)$ in Theorem 1 and expressions for $f(x, y \mid u)$ and $f(x \mid u)$, for $u>0$, in Theorem 2. Section 2 contains Theorem 1 together with its proof and some discussion. Simplifications in the case of phasetype interclaim arrival times are given in Proposition 1 in Section 2.1. Theorem 2 is stated and proved in Section 3. In Section 4 we consider the case where the claim-size distribution is heavy-tailed, while in Section 5 we establish conditions for the joint moments of $U(T-)$ and $|U(T)|$ to be finite. Finally, Section 6 contains some concluding remarks.

\section{The joint density for zero initial surplus}

Our approach for obtaining the joint density of $U(T-)$ and $|U(T)|$ when $u$ is 0 is via the random walk associated with the surplus process $\{U(t): t \geq 0\}$. To simplify the notation, we assume, from now on and without loss of generality, that $c=1$ in the model. Then, for $i=1,2,3, \ldots$, let $Y_{i}=T_{i}-X_{i}$ and, for $n \in \mathbb{N}$,

$$
S_{n}=\sum_{i=1}^{n} Y_{i}
$$

The link between the continuous-time process and the random walk is provided by the observation that ruin may occur only at claim instants, so that, for instance, the probability of ruin can be expressed as $\psi(u)=\mathrm{P}\left(\inf S_{n}<-u: n=1,2, \ldots\right)$.

Now let $G_{+}$and $G_{-}$be the distributions of the (strict) ascending ladder height and the (weak) descending ladder height, respectively, of the random walk $\left\{S_{n}: n=1,2, \ldots\right\}$. For definitions of these, as well as various other aspects of random walk theory and its connection to risk theory, we refer the reader to the books by Feller (1971, Chapter XII), Asmussen (1987, Chapter VII), and Embrechts et al. (1997, Chapter 8). We note, in particular, that, provided that the step distribution of the random walk has a nonzero finite first moment, one of $G_{+}$and $G_{-}$ is a proper probability distribution and the other is defective. Here, we assume, throughout, that

$$
0<\mathrm{E}\left(Y_{1}\right)<\infty
$$

on noting that if $\mathrm{E}\left(Y_{1}\right) \leq 0$, ruin is certain to occur. Under this assumption, the ascending ladder height distribution $G_{+}$is proper, while $G_{-}$is defective. Note that $G_{+}$is concentrated on $(0, \infty)$ and $G_{-}$on $(-\infty, 0]$ and that they are related via the Wiener-Hopf equation; see, e.g. Feller (1971, Chapter XII). In our first main result, below, we obtain the joint density $f(x, y \mid 0)$ for the Sparre Andersen model. It is worth noting that, for any value $u$ of the initial surplus, the following important formula holds:

$$
f(x, y \mid u)=f(x \mid u) \frac{p(x+y)}{1-P(x)} .
$$

For the classical model, this was derived by Dufresne and Gerber (1988). It is easy to see that the formula also holds for the Sparre Andersen model that we consider here. Letting $u=0$ in (3), we see that in order to obtain $f(x, y \mid 0)$, it is sufficient to find $f(x \mid 0)$.

When we consider the probability of ruin and the deficit at ruin, it is a well-known fact that the descending ladder height distribution of the random walk plays a key role. For instance, let $\mu_{-}$be the measure associated with the distribution $G_{-}$on $(-\infty, 0]$ and define a new measure $\check{\mu}_{-}$by

$$
\check{\mu}_{-}\{A\}=\mu_{-}\{x:-x \in A\}
$$


for any measurable set $A \subset[0, \infty)$. Let $\check{G}_{-}(x)=\check{\mu}_{-}([0, x])$ be the distribution function associated with $\check{\mu}_{-}$. Then it holds that

$$
1-\psi(u)=(1-\psi(0)) \sum_{m=0}^{\infty} \check{G}_{-}^{\star m}(u),
$$

where $\check{G}_{-}^{\star m}$ denotes the $m$-fold Lebesgue-Stieltjes convolution power of $\check{G}_{-}$. In the sequel, we shall consider convolution as a more general operator, defined between a function $q$, which is bounded on finite intervals, and a nondecreasing, right continuous function $F$. Then their convolution, denoted by $q \star F$ is defined by

$$
(q \star F)(x)=\int q(x-t) \mathrm{d} F(t) .
$$

Note that, for $m=0$ in (4), $\check{G}_{-}^{\star 0}(x)=\mathbf{1}_{[0, \infty)}(x)$, the indicator function on the nonnegative half-axis.

Furthermore, let

$$
F_{1}(u, y)=\mathrm{P}\left(\left|U_{T}\right| \leq y \mid U_{0}=u\right)
$$

be the distribution function of the deficit at ruin; see Gerber et al. (1987). Then, for $u=0$, it is well known (see, e.g. Willmot (2002)) that

$$
F_{1}(0, y)=\check{G}_{-}(y) .
$$

The next theorem, which generalises the key formula, (2), for $\delta=0$, shows that when we consider the surplus prior to ruin, the role played by the descending ladder height is now undertaken by the ascending ladder height of the random walk $\left\{S_{n}: n=1,2, \ldots\right\}$.

Theorem 1. For $x \geq 0$, let

$$
U_{+}(x)=\sum_{k=0}^{\infty} G_{+}^{\star k}(x)
$$

be the renewal function associated with the ascending ladder height of the random walk $\left\{S_{n}: n=1,2, \ldots\right\}$. Then the joint density of the surplus before ruin and the deficit at ruin for zero initial surplus in the Sparre Andersen model is given by

$$
f(x, y \mid 0)=p(x+y) \int_{[0, x]} k(x-z) \mathrm{d} U_{+}(z) .
$$

Proof. As noted above, it suffices to concentrate on the density $f(x \mid 0)$ of the surplus prior to ruin for zero initial surplus and this is what we do next.

First, for $x>0$ and $n=1,2, \ldots$, let

$$
H_{n}(x)=\mathrm{P}\left(S_{1}>0, S_{2}>0, \ldots, S_{n-1}>0, S_{n} \in(0, x]\right)
$$

and define

$$
H(x)=\sum_{n=1}^{\infty} H_{n}(x) .
$$

Recall that $T$ is the time to ruin in the model, and let $\kappa$ be the time of the claim prior to ruin. We note that $\kappa$ could be 0 , if ruin occurs with the first claim. Considering this separately and, 
if this is not the case, asking how many claims there will be until ruin and how long is the time interval between $\kappa$ and $T$, we obtain

$$
\begin{aligned}
f(x \mid 0) & =k(x)[1-P(x)]+\sum_{n=1}^{\infty} \int_{0}^{x} k(x-t)[1-P(x)] \mathrm{d} H_{n}(t) \\
& =[1-P(x)]\left(k(x)+\int_{[0, x]} k(x-t) \mathrm{d} H(t)\right) .
\end{aligned}
$$

The factor $[1-P(x)]$ arises since we ask that, as soon as the surplus reaches $x$, there is a claim causing ruin, and this is independent of anything prior to that.

Furthermore, using the duality lemma for random walks (Feller 1971, Chapter XII), we obtain

$$
\begin{aligned}
H(x) & =\sum_{n=1}^{\infty} \mathrm{P}\left(S_{1}>0, S_{2}>0, \ldots, S_{n-1}>0, S_{n} \in(0, x]\right) \\
& =\sum_{n=1}^{\infty} \mathrm{P}\left(S_{n}>S_{1}, S_{n}>S_{2}, \ldots, S_{n}>S_{n-1}, S_{n} \in(0, x]\right) .
\end{aligned}
$$

So, inside the bracket on the right-hand side we have the probability that $S_{n}$ is an ascending ladder height for the random walk and lies inside $(0, x]$. Therefore, summing over all $n$, we obtain the expected number of ladder heights in that interval. Since the distribution of the ascending ladder height is $G_{+}$, we have therefore shown that

$$
H(x)=\sum_{n=1}^{\infty} G_{+}^{\star k}(x) .
$$

From this we see that $H(x)=U_{+}(x)-\mathbf{1}_{[0, \infty)}(x)$, i.e. the measure associated with $H$ is the restriction of the renewal measure $U_{+}$on $(0, \infty)$. In view of this, (9) yields

$$
f(x \mid 0)=[1-P(x)] \int_{[0, x]} k(x-z) \mathrm{d} U_{+}(z),
$$

and the result of Theorem 1 now follows from (3).

Note that the only potential difficulty in the above formula concerns $U_{+}$, which may not be available analytically. In Section 2.1 we derive some explicit expressions for the density $f(x, y \mid 0)$ when the distribution $K$ belongs to the phase-type family.

We now make a few remarks on the implications of Theorem 1. First, we note that, since we assume that the distribution of times between claims, $K$, has a density, the ascending ladder height distribution is also absolutely continuous and from (10) it follows that $H$ has a density, say $h$, which is also a density for $U_{+}$on $(0, \infty)$. Therefore, we see that the result of Theorem 1 can be written in terms of this density as follows:

$$
f(x \mid 0)=[1-P(x)]\left(k(x)+\int_{0}^{x} k(x-t) h(t) \mathrm{d} t\right) .
$$

Another simple observation is that in the classical risk model, when $T_{i}$ is exponential with parameter $\lambda$, it is well known that $G_{+}$also has an exponential distribution. This implies that 
$h(x)=\lambda$ for all $x \geq 0$. Then (12) yields

$$
f(x \mid 0)=[1-P(x)]\left(\lambda \mathrm{e}^{-\lambda x}+\lambda \int_{0}^{x} \lambda \mathrm{e}^{-\lambda t} \mathrm{~d} t\right)=\lambda[1-P(x)],
$$

which, when scaling back for $c$ and using (3), agrees with the key formula, (2), of Gerber and Shiu (1997).

Next, Dufresne and Gerber (1988) have proved that for the classical model it holds that

$$
f(x, y \mid 0)=f(y, x \mid 0),
$$

a symmetry which is obvious from (2); see also Dickson (1992) for a discussion on this. This symmetry, as can be seen from Theorem 1, is no longer valid in the Sparre Andersen model.

\subsection{Explicit expressions for phase-type distributions}

Phase-type distributions have a central position in a variety of applied probability models. Following the pioneering work of Neuts (1981), recent work by Asmussen and coauthors, most of which is contained in Asmussen (2000, Chapter VIII), has demonstrated that, if the solution to a particular problem can be found explicitly in the exponential case, then an explicit solution in matrix form can be found if the underlying distribution belongs to the phase-type (PH) family. For details and the main properties of distributions in that family we refer the reader to the books mentioned above; here we merely note that a distribution $B$ is said to be of phase-type if it represents the time until absorption in a terminating Markov process with state space $\{0\} \cup E$, where 0 is the absorbing state and $E$ is a finite set of transient states, and with time-homogeneous transition rates. We write $(\pi, \mathbb{T})$ for the PH representation of $B$, where the row vector $\pi$ and the square matrix $\mathbb{T}$ are such that $B(x)=1-\pi \mathrm{e}^{\mathbb{T} x} e_{0}$, where $e_{0}$ is a column vector of $1 \mathrm{~s}$. Furthermore, if $B$ has density $b$, then $b(x)=\pi \mathrm{e}^{\mathbb{T} x} t_{0}$, where $t_{0}=-\mathbb{T} e_{0}$ is the vector of exit rates in the associated Markov jump process; see, for example, Asmussen (2000, Chapter VIII).

Drekic et al. (2004) proved that in the Sparre Andersen model the distribution of the deficit at ruin is phase-type provided that the claim size distribution is phase-type. To parallel this, we obtain a $\mathrm{PH}$ representation for the surplus prior to ruin when the interclaim times have a $\mathrm{PH}$ distribution $K$. Our present setup generalises that of Gerber and Shiu (2005), in which they consider the case where interclaim times are distributed as a finite sum of independent exponential random variables with possibly different parameters, i.e. $K$ belongs to a particular subclass of phase-type distributions. In contrast, our result below applies when $K$ has a general phase-type distribution.

Before stating our result, note that in the remainder of this paper, we shall often make use of the following conditions on the distributions $K$ and $P$ of the model.

(i) The distribution $K$ of interclaim times has a density $k$ which is bounded and such that $\lim _{x \rightarrow \infty} k(x)=0$.

(ii) The distribution $P$ of claim sizes has unbounded support.

In the sequel, we refer to these as conditions (i) and (ii), respectively. Under condition (ii), $P(x)<1$ for all $x$ and we may define the function $v(x)$ by

$$
v(x)=\frac{f(x \mid 0)}{1-P(x)} .
$$


We see from Theorem 1 that

$$
v(x)=\int_{[0, x]} k(x-z) \mathrm{d} U_{+}(z)
$$

Proposition 1. Assume that in the Sparre Andersen model the distribution $K$ of interclaim times is a phase-type distribution with representation $(\pi, \mathbb{T})$ and state space $E_{0}$, while the distribution $P$ of claims is an arbitrary distribution with an infinite right-end point (so that condition (ii) is satisfied). Then, the function $v(x)$ in (13) can be expressed as

$$
v(x)=\pi \exp \left(\left(\mathbb{T}+t_{0} \pi_{+}\right) x\right) t_{0},
$$

where $t_{0}=-\mathbb{T} e_{0}$ and $\pi_{+}$is a row vector satisfying

$$
\pi_{+}=\pi \hat{p}\left[-\left(\mathbb{T}+t_{0} \pi_{+}\right)\right] .
$$

Proof. Applying Theorem 2.1 of Asmussen (1992) with $U, T$, and $X$ there replaced by our $T_{1}, X_{1}$, and $Y_{1}$, respectively, we see that, under the assumptions of the proposition, the ascending ladder height distribution $G_{+}$has PH representation $\left(\pi_{+}, \mathbb{T}+t_{0} \pi_{+}\right)$, where $\pi_{+}$satisfies (15). From (2.6) of Asmussen (1992) the renewal density associated with $G_{+}$is given by

$$
u_{+}(x)=\pi_{+} \exp \left(\left(\mathbb{T}+t_{0} \pi_{+}\right) x\right) t_{0}
$$

From (14), we see that $v(x)$ is a density of $K \star U_{+}$. Asmussen (1992) obtained an expression for the density of $K \star U_{+}$in the proof of his Corollary 2.3. We follow Asmussen and argue as in the proof of Corollary 2.2 of Asmussen (1992). We think of $K \star U_{+}$as being represented by a particle which initially (the $K$ part) starts according to $\pi$, moves according to $\mathbb{T}$, and then exits according to $t_{0}$, to be restarted (the renewal part) according to $\pi_{+}$and then moved according to $\mathbb{T}+t_{0} \pi_{+}$, i.e. in exactly the same way as is described for the first part. Thus, we find that $K \star U_{+}$is represented by the density

$$
\pi \exp \left(\left(\mathbb{T}+t_{0} \pi_{+}\right) x\right) t_{0}
$$

which is what Asmussen (1992) obtained in the proof of his Corollary 2.3.

Now we compare our results with those of Badescu et al. (2005b), who used techniques from fluid queues to analyse risk processes with claims arriving in a Markovian arrival process and with claim sizes having a phase-type distribution. Both their model and ours include as a special case the Sparre Andersen model with phase-type interclaim and claim size distributions. We show that for this $\mathrm{PH} / \mathrm{PH}$ model, the expressions for $f(x \mid 0)$ in their paper and here are the same. In Proposition 2, suppose that $K$ is of order $m$ and, in addition, assume that the claims are $\mathrm{PH}$ of order $n$ with representation $(\gamma, M)$. Let $g_{0}=-M e_{0}$. In this setup, equation (4) of Badescu et al. (2005b) can be written in our notation as

$$
f(x \mid 0)=\pi \mathrm{e}^{\mathbb{S} x} t_{0} \gamma \mathrm{e}^{M x} e_{0}=\pi \mathrm{e}^{\mathbb{S} x} t_{0}(1-P(x)),
$$

where $\mathrm{e}^{\mathbb{S} x}$ is the $m \times m$ matrix whose $(i, j)$ th element is equal to the expected number of visits of the surplus process to level $x$, phase $j$, before the first return to 0 , given that the initial interclaim time starts in phase $i$. Comparing this with (14), we aim to show that our $v(x)$ is the same as $\pi \mathrm{e}^{\mathbb{S} x} t_{0}$. 
Let $J_{t}$ be the phase at time $t$ in the fluid process corresponding to the surplus process (see Badescu et al. (2005a)), and define

$$
H_{n, i}(x)=\mathrm{P}\left(S_{1}>0, \ldots, S_{n-1}>0, S_{n} \in(0, x] \mid J_{0}=i\right),
$$

so that

$$
\sum_{i=1}^{m} H_{n, i}(x) \pi_{i}=H_{n}(x)
$$

(see (7)). Let $\left\{J_{x}^{(T)}\right\}_{x \geq 0}$ be the (terminating) Markov phase process for an interclaim time, so that $J_{x}^{\left(T_{1}\right)}=j$ means that $T_{1}>x$ and that the phase is $j$ when $T_{1}=x$. For $i, j \in\{1, \ldots, m\}$, we have

$$
\begin{aligned}
\left(\mathrm{e}^{\mathbb{S} x}\right)_{i j}= & \mathrm{E}\left(\mathbf{1}\left(J_{x}^{\left(T_{1}\right)}=j\right) \mid J_{0}=i\right) \\
& +\mathrm{E}\left(\sum_{n=2}^{\infty} \mathbf{1}\left(S_{1}>0, \ldots, S_{n-2}>0, S_{n-1} \in(0, x], J_{x-S_{n-1}}^{\left(T_{n}\right)}=j\right) \mid J_{0}=i\right),
\end{aligned}
$$

where we write $\mathbf{1}(A)$ for the indicator function of the event $A$. The first term on the right-hand side is $\left(\mathrm{e}^{\mathbb{T} x}\right)_{i j}$. By considering the phase with which $T_{n}$ starts, the second term can be seen to be

$$
\sum_{n=2}^{\infty} \sum_{k=1}^{m} \int_{(0, x]} \pi_{k}\left(\mathrm{e}^{\mathbb{T}(x-y)}\right)_{k j} H_{n-1, i}(\mathrm{~d} y) .
$$

This means that the Badescu et al. (2005b) expression is

$$
\begin{aligned}
\pi \mathrm{e}^{\mathbb{S} x} t_{0} & =\pi \mathrm{e}^{\mathbb{T} x} t_{0}+\sum_{n=2}^{\infty} \sum_{i=1}^{m} \sum_{j=1}^{m} \sum_{k=1}^{m} \int_{(0, x]} \pi_{k}\left(\mathrm{e}^{\mathbb{T}(x-y)}\right)_{k j}\left(t_{0}\right)_{j} \pi_{i} H_{n-1, i}(\mathrm{~d} y) \\
& =k(x)+\sum_{n=2}^{\infty} \sum_{i=1}^{m} \int_{(0, x]} k(x-y) \pi_{i} H_{n-1, i}(\mathrm{~d} y) \\
& =k(x)+\sum_{n=2}^{\infty} \int_{(0, x]} k(x-y) H_{n-1}(\mathrm{~d} y)
\end{aligned}
$$

This yields the same expression for $f(x \mid 0)$ as we obtained above in (8), so our results coincide with those of Badescu et al. (2005b) for the special case of the PH/PH Sparre Andersen model.

Note that Badescu et al. (2005b) considered a different model in general from the model we consider in this paper. We consider the Sparre Andersen model with general interclaim time distribution and general claim size distribution, but with the strict independence assumptions of the Sparre Andersen model, while Badescu et al. (2005b) considered only phase-type claim sizes throughout, with a Markovian claim arrival process, which allowed for dependency between interclaim times and claims.

The condition for the interclaim time distribution $K$ to be phase-type is not necessary if we are to obtain an explicit expression for the ascending ladder height distribution $G_{+}$. As an example, assume that the distribution $P$ of claim sizes is exponential with parameter $\theta$, while $K$ is arbitrary. Let $G$ be the distribution of the variable $Y_{i}=T_{i}-X_{i}$ and $g$ be a density for $G$. Since

$$
G(x)=\int_{[0, \infty)} K(x+y) \mathrm{d} P(y),
$$


it follows that $G(x)=a \mathrm{e}^{\theta x}$ for $x<0$ and for some $a>0$. Furthermore, it is well known that in this case $G_{-}$has a (defective) density $g_{-}(x)=b_{0} \mathrm{e}^{\theta x}$ for $x<0$. Then, arguing as in Asmussen (1987, p. 203), but interchanging the role of the axes since Asmussen (1987) considers a random walk with negative drift, we find that the ascending ladder height distribution $G_{+}$is given by

$$
G_{+}(x)=1-\frac{1}{1-r_{0}}\left(\bar{G}(x)-r_{0} \mathrm{e}^{\eta x} \int_{[x, \infty)} \mathrm{e}^{-\eta z} \mathrm{~d} G(z)\right), \quad x \geq 0 .
$$

Here $\bar{G}(x)=1-G(x), r_{0}=b_{0} \theta^{-1}$, and $\eta=\theta-b_{0}$. By differentiating the distribution $G_{+}$, above, we also obtain its density, $g_{+}(x)$, which is given by

$$
g_{+}(x)=g(x)+r_{0}\left(1-r_{0}\right)^{-1} \eta \mathrm{e}^{\eta x} \int_{[x, \infty)} \mathrm{e}^{-\eta z} \mathrm{~d} G(z) .
$$

\section{The joint density for arbitrary initial surplus}

Next, we look for an expression of the joint density for arbitrary initial surplus $u$. For the classical risk model it is known that the following is true:

$$
f(x, y \mid u)= \begin{cases}f(x, y \mid 0) \frac{1-\psi(u)}{1-\psi(0)}, & x>u>0 \\ f(x, y \mid 0) \frac{\psi(u-x)-\psi(u)}{1-\psi(0)}, & 0<x \leq u\end{cases}
$$

see Dickson (1992) and Gerber and Shiu (1998). This does not hold for the Sparre Andersen model. Instead, for this model, Dickson and Drekic (2004) have argued that the joint density $f(x, y \mid u)$ is readily available if $f(x, y \mid 0)$ is known. To support this argument, they have shown that the following holds:

$$
f(x, y \mid u)=\frac{p(x+y)}{\zeta(0)} \int_{\left[(u-x)_{+}, u\right]} \frac{f(x-u+z \mid 0)}{\bar{P}(x-u+z)} \mathrm{d} \zeta(z),
$$

where $\zeta(u)=1-\psi(u)$ denotes the probability of nonruin in the Sparre Andersen model, $\bar{P}(x)=1-P(x)$, and $(u-x)_{+}=\max \{0, u-x\}$.

The first part of the next result is an easy consequence of Theorem 1, while the second part shows that (17) is asymptotically correct for the Sparre Andersen model.

Theorem 2. (i) In the Sparre Andersen model, the joint density of the surplus before and after ruin is given by

$$
f(x, y \mid u)=\frac{p(x+y)}{\zeta(0)} \int_{\left[(u-x)_{+}, u\right]}\left(k \star U_{+}\right)(x-u+z) \mathrm{d} \zeta(z),
$$

while the density of the surplus before ruin for initial surplus $u$ is given by

$$
f(x \mid u)=\frac{1-P(x)}{\zeta(0)} \int_{\left[(u-x)_{+}, u\right]}\left(k \star U_{+}\right)(x-u+z) \mathrm{d} \zeta(z) .
$$

(ii) Assume that conditions (i) and (ii) hold. Then, as $x \rightarrow \infty$, and for all values of $u$, it holds that

$$
\lim _{x \rightarrow \infty} \frac{f(x \mid u)}{1-P(x)}=\frac{1-\psi(u)}{\mathrm{E}\left(Y_{1}\right)} .
$$


Proof. Substituting (11) into (18) immediately yields (19) and then (20) follows from (3). This establishes the first part of Theorem 2 .

For the second part, we first obtain the asymptotic behaviour of $f(x \mid 0)$ for large $x$. Theorem 1 shows that $v(x)$ (defined in (13)) is given by (14), which in turn shows that $v$ satisfies the following proper (i.e. nondefective) renewal equation:

$$
v(x)=k(x)+\int_{[0, x]} v(x-z) \mathrm{d} G_{+}(z) .
$$

Note that, since $K$ has a density, this implies that the step distribution of the random walk $S_{n}$, i.e. the distribution of the random variable $Y_{1}$, has a density. This, in turn, implies that the distribution $G_{+}$is absolutely continuous; see Feller (1971, Chapter XII). Now employing a version of the key renewal theorem, which is Corollary 1.3 in Chapter VI of Asmussen (1987), we deduce, from (22), that

$$
\lim _{x \rightarrow \infty} v(x)=\frac{1}{\mu_{0}}
$$

where

$$
\mu_{0}=\int x \mathrm{~d} G_{+}(x) .
$$

However, since $\mathrm{E}\left(Y_{1}\right)$ denotes the mean step size for the random walk $\left\{S_{n}: n=1,2, \ldots\right\}$, it is well known (see, for instance, Asmussen (1987, Chapter VII)) that

$$
\int x \mathrm{~d} G_{+}(x)=\left(1-G_{-}(\infty)\right)^{-1} \mathrm{E}\left(Y_{1}\right)
$$

and, since $G_{-}(\infty)=\psi(0)$, we obtain

$$
\lim _{x \rightarrow \infty} \frac{f(x \mid 0)}{1-P(x)}=\frac{1-\psi(0)}{\mathrm{E}\left(Y_{1}\right)},
$$

which gives the asymptotic behaviour for large $x$.

Next, we note that the distribution function $\zeta$, which corresponds to the distribution of the maximal aggregate loss in the model, has a density, say $q$, on $(0, \infty)$.

For $u \leq x,(20)$ then gives

$$
f(x \mid u)=(1-P(x))\left(k \star U_{+}\right)(x-u)+\frac{1-P(x)}{\zeta(0)} \int_{0}^{u}\left(k \star U_{+}\right)(x-u+z) q(z) \mathrm{d} z .
$$

Dividing both sides by $1-P(x)$, and noting the definition of the function $v(x)$, we thus obtain

$$
\frac{f(x \mid u)}{1-P(x)}=v(x-u)+\frac{1}{\zeta(0)} \int_{0}^{u} v(x-u+z) q(z) \mathrm{d} z
$$

From (23), we see that the first term above tends to $\mu_{0}^{-1}$, while for the second term we use the dominated convergence to deduce that this converges to

$$
\frac{\zeta(u)-\zeta(0)}{\mu_{0} \zeta(0)}
$$

Putting these two results together, and using the fact that $\mu_{0}=\mathrm{E}\left(Y_{1}\right) / \zeta(0)$, completes the proof of the theorem. 
Remarks. (i) If the distribution $P$ of claim sizes is concentrated on a compact set, then the asymptotic behaviour of $f(x \mid 0)$ is trivial. For in this case, there is an $m>0$ such that $P(m)=1$. It is then probabilistically obvious that $f(x \mid u)=0$ for all $x>m$ and for any value $u$ of the initial surplus.

(ii) In the standard version of the key renewal theorem (see, e.g. Feller (1971, Chapter XI) or Asmussen (1987, Chapter IV)), a key role is played by direct Riemann integrability. We have used an alternative version merely to avoid reference to this; however, we note that necessary and sufficient conditions for a function to be directly Riemann integrable (d.R.i.) are given in Asmussen (1987, Section IV.4). For instance, since $k$ is a probability density, if it is a nonincreasing function then it is automatically d.R.i., so that the key renewal theorem applies again.

(iii) Furthermore, note that in the proof of Theorems 1 and 2, we made no assumption that the distribution $P$ of claims has a density. It is worth noting that (11), for instance, is valid for any claim size distribution. The results of Theorems 1 and 2, however, do not hold as they stand, but need a slight modification if $P$ is not absolutely continuous. The most important case then is when $P$ is discrete, and assume, for simplicity, that it is concentrated on the set of positive integers; see, e.g. Picard and Lefèvre (1998), (2003) for various aspects of the classical risk model under this assumption. When we consider the joint density, $f(x, y \mid 0)$, it is clear that this equals 0 unless $(x+y) \in \mathbb{N}$. For such values of $x$ and $y$, it is easy to see that (6) still holds provided that we interpret $p(x+y)$ in (6) as the probability that a claim equals $x+y$.

When $x<u$, the density of the surplus before ruin in (20) becomes

$$
f(x \mid u)=\frac{1-P(x)}{\zeta(0)} \int_{[u-x, u]}\left(k \star U_{+}\right)(x-u+z) \mathrm{d} \zeta(z),
$$

and if $q$ is a density for the measure $\zeta$, as above, this reduces to

$$
f(x \mid u)=\frac{1-P(x)}{\zeta(0)} \int_{u-x}^{u}\left(k \star U_{+}\right)(x-u+z) q(z) \mathrm{d} z .
$$

Setting $w=u-z$, we obtain

$$
f(x \mid u)=\frac{1-P(x)}{\zeta(0)} \int_{0}^{x}\left(k \star U_{+}\right)(x-w) q(u-w) \mathrm{d} w .
$$

Now, for $u \geq 0$, let a function $q_{u}$ be defined by $q_{u}(w)=q(u-w)$ for $0 \leq w<u$ and $q_{u}(w)=0$ otherwise. Then the last expression takes the following simpler form:

$$
f(x \mid u)=\frac{1-P(x)}{\zeta(0)} \int_{0}^{x}\left(k \star U_{+}\right)(x-w) q_{u}(w) \mathrm{d} w,
$$

which expresses the surplus prior to ruin as the product of a function of $x$ and the convolution between two quantities which are in many cases (e.g. in the phase-type case) easy to obtain.

Furthermore, note that, from (3) and Theorem 2(ii) it easily follows that

$$
\lim _{x \rightarrow \infty} \frac{f(x, y \mid u)}{f(x, y \mid 0)}=\frac{1-\psi(u)}{1-\psi(0)} .
$$

This shows that (17) holds asymptotically for the Sparre Andersen model. 


\section{Subexponential distributions}

An important class of distributions for modelling claim size distributions with a heavy tail is the class of subexponential distributions; see e.g. Embrechts et al. (1997). A classical result in this area is due to Embrechts and Veraverbeke (1982); more precisely, let

$$
P_{\mathrm{e}}(x)=\frac{1}{\mathrm{E}\left(X_{1}\right)} \int_{0}^{x}(1-P(t)) \mathrm{d} t
$$

be the integrated tail distribution associated with the claim size distribution $P$ in the Sparre Andersen model and $\rho=\left\|G_{-}\right\|$be the total mass of the defective distribution $G_{-}$, the descending ladder height distribution associated with the random walk $\left\{S_{n}: n=1,2 \ldots\right\}$. Then, under the assumption that $P_{\mathrm{e}}$ is subexponential, it holds that

$$
\psi(x) \sim \frac{\rho\left(1-P_{\mathrm{e}}(x)\right)}{1-\rho} .
$$

Note that the symbol $\sim$ here, and in the sequel, means that the ratio of the two functions tends to 1 as $x \rightarrow \infty$.

Based on Theorem 2, our next result establishes a condition for the distribution function of the surplus prior to ruin to belong to the class of subexponential distributions, which we denote by $\&$. First note that, if $F(x \mid u)$ denotes the distribution function of the surplus prior to ruin,

$$
F(x \mid u)=\mathrm{P}(U(T-) \leq x \mid U(0)=u),
$$

so that $F(\cdot \mid u)$ has density $f(\cdot \mid u)$, then $F$ is a defective distribution. To this end, we consider the conditional distribution of the surplus prior to ruin, given that ruin occurs, namely

$$
F_{2}(x \mid u)=\mathrm{P}(U(T-) \leq x \mid T<\infty, U(0)=u),
$$

which is a proper cumulative distribution function (CDF) for any value of the initial surplus $u$. Furthermore, $F_{2}$ is related to $F$ by

$$
F_{2}(x \mid u)=\frac{F(x \mid u)}{\psi(u)} \quad \text { for } x, u \geq 0 .
$$

Then we have the following proposition.

Proposition 2. Assume that in the Sparre Andersen model condition (i) holds and that the distribution $P_{\mathrm{e}}$ is in \&. Then $F_{2}(x \mid u)$ also belongs to \&.

Proof. For $x \geq 0$, let $p_{\mathrm{e}}(x)=[1-P(x)] / \mathrm{E}\left(X_{1}\right)$, so that $p_{\mathrm{e}}$ is a density for the integrated tail distribution $P_{\mathrm{e}}$ and let $\theta_{0}=\mathrm{E}\left(Y_{1}\right) / \mathrm{E}\left(X_{1}\right)$. Then we observe that (21) can be written as

$$
\lim _{x \rightarrow \infty} \frac{f(x \mid u)}{p_{\mathrm{e}}(x)}=\frac{1-\psi(u)}{\theta_{0}} .
$$

This shows that $f(x \mid u) \sim A p_{e}(x)$, where $A=A(u)=(1-\psi(u)) / \theta_{0}$. By a simple integration of (26) and using (25) it is then easy to see that

$$
1-F_{2}(x \mid u) \sim \frac{A}{\psi(u)}\left(1-P_{\mathrm{e}}(x)\right),
$$

i.e. the distributions $F_{2}(\cdot \mid u)$ and $P_{\mathrm{e}}$ are tail-equivalent for every fixed $u \geq 0$. The result now follows from Corollary 1.10 in Chapter IX of Asmussen (2000). 
Remarks. (i) Note that in the proof of Proposition 2 we have used (21), which in turn requires both conditions (i) and (ii) to hold. However, it is immediate that the condition for $P_{\mathrm{e}}$ to be in $\delta$ is stronger than condition (ii); therefore, condition (ii) is not needed in the statement of the proposition.

(ii) We note that if the claim size distribution $P$ itself is in $\delta$, then under some further mild conditions (see Klüppelberg (1988)), $P_{\mathrm{e}}$ is in $\$$, so that Proposition 2 applies.

A class which generalises the class of subexponential distributions is the following. A distribution function $F$ on $[0, \infty)$ belongs to the class $\delta(\gamma)$ for $\gamma \geq 0$ if it satisfies the following conditions:

(a) $\lim _{x \rightarrow \infty} \overline{F^{\star 2}}(x) / \bar{F}(x)=2 \tilde{F}(\gamma)<\infty$,

(b) $\lim _{x \rightarrow \infty} \bar{F}(x-y) / \bar{F}(x)=\mathrm{e}^{\gamma y}$ for all real $y$,

where $\tilde{F}$ is the moment generating function of $F$. For $\gamma=0$, it is well known (see, for example, Klüppelberg (1988)) that (a) implies (b), so that $\&(0)$ coincides with $\&$. For $\gamma>0$, we have the following proposition, which gives a condition for the CDF of the surplus prior to ruin to be in the class $\delta(\gamma)$.

Proposition 3. Let $\gamma>0$ and assume that $P$ is the claim size distribution and $F(\cdot \mid u)$ is the $C D F$ of the surplus prior to ruin in the Sparre Andersen model. Then, for any $u \geq 0, P \in \delta(\gamma)$ if and only if $F_{2}(x \mid u)$ is in $\delta(\gamma)$.

Proof. It follows from the results in Klüppelberg (1989), see also Cheng et al. (2002), that $P \in \delta(\gamma)$ if and only if $P_{\mathrm{e}} \in \delta(\gamma)$. The result now follows from (27) and the well-known fact that the class $\delta(\gamma)$ is also closed under tail-equivalence; see, e.g. Teugels (1975).

\section{Joint moments of the surplus prior to ruin and the deficit at ruin}

In this section we establish conditions for finiteness of moments for the joint distribution of the surplus prior to ruin, $U(T-)$, and the deficit at ruin, $|U(T)|$. For the classical risk model, Lin and Willmot (2000) have established explicit expressions for the joint moments of these two quantities, which are, however, rather cumbersome as they depend on recursive formulae for such moments and require knowledge of the ruin probability $\psi(u)$.

Here, the following proposition gives conditions for the finiteness of these moments in the Sparre Andersen model. As usual, we proceed by establishing the result for $u=0$ first.

Proposition 4. Assume that in the Sparre Andersen model the claim-size distribution $P$ has finite moments of order $r$ for some $r>1$ and that the interclaim distribution $K$ has a bounded density $k$.

Let $G$ be the distribution of the variable $Y_{i}=T_{i}-X_{i}$, so that $G$ is the step distribution of the random walk $\left\{S_{n}: n=1,2, \ldots\right\}$ and assume that $G$ has a finite first moment. Then, for any nonnegative integers $m$ and $n$ such that $m+n<r-1$, it holds that

$$
\int_{0}^{\infty} \int_{0}^{\infty} y^{n} x^{m} f(x, y \mid 0) \mathrm{d} x \mathrm{~d} y<\infty .
$$

Proof. We first make the following claim: for all $m$ and $n$ such that $m+n<r-1$, it holds that

$$
I_{0}:=\int_{0}^{\infty} \int_{0}^{\infty} y^{n} x^{m} p(x+y) \mathrm{d} x \mathrm{~d} y<\infty .
$$


For $m=0$, note that the claim is true in view of the moment conditions for the distribution $P$ in the statement. To prove the claim for positive $m$, we first calculate the inner integral of $I_{0}$. Using integration by parts, we have

$$
\int_{0}^{\infty} y^{n} x^{m} p(x+y) \mathrm{d} x=y^{n}\left[-x^{m} \bar{P}(x+y)\right]_{0}^{\infty}+m y^{n} \int_{0}^{\infty} x^{m-1} \bar{P}(x+y) \mathrm{d} x .
$$

Since the distribution $P$ has finite moments of order $r$, the first term on the right-hand side is 0 . Furthermore, it is readily checked that the associated tail, $\bar{P}=1-P$, satisfies, for all nonnegative $x$ and $y$,

$$
\bar{P}(x+y) \leq C_{0}(1+x+y)^{-r}
$$

for some positive constant $C_{0}$. This gives

$$
\begin{aligned}
\int_{0}^{\infty} y^{n} x^{m} p(x+y) \mathrm{d} x & \leq m C_{0} y^{n} \int_{0}^{\infty} x^{m-1}(1+x+y)^{-r} \mathrm{~d} x \\
& \leq m C_{0} y^{n} \int_{0}^{\infty}(1+x+y)^{-r+m-1} \mathrm{~d} x \\
& =m(r-m)^{-1} C_{0} y^{n}(1+y)^{-r+m} .
\end{aligned}
$$

Then, integrating with respect to $y$, and since $m+n-r<-1$, we immediately obtain

$$
\int_{0}^{\infty} y^{n}(1+y)^{-r+m} \mathrm{~d} y<\infty
$$

and this proves the claim in (29).

Now considering the joint moments of the density, $f(x, y \mid 0)$, we use the expression for $f(x, y \mid 0)$ from Theorem 1 to deduce that

$$
\int_{0}^{\infty} \int_{0}^{\infty} y^{n} x^{m} f(x, y \mid 0) \mathrm{d} x \mathrm{~d} y=\int_{0}^{\infty} \int_{0}^{\infty} y^{n} x^{m} p(x+y) \int_{0}^{x} k(x-t) \mathrm{d} U_{+}(t) \mathrm{d} x \mathrm{~d} y .
$$

Next, we employ Stone's decomposition theorem (see, for instance, Asmussen (1987, Chapter VI)), which asserts that $U_{+}$can be written as $U_{+}=U_{+}^{(1)}+U_{+}^{(2)}$, where $U_{+}^{(1)}$ has a bounded continuous density $h_{1}(t) \rightarrow \mu_{0}^{-1}$ and $U_{+}^{(2)}$ is a finite measure. Consequently, we obtain

$$
\begin{aligned}
\int_{0}^{\infty} \int_{0}^{\infty} y^{n} x^{m} f(x, y \mid 0) \mathrm{d} x \mathrm{~d} y= & \int_{0}^{\infty} \int_{0}^{\infty} y^{n} x^{m} p(x+y) \int_{0}^{x} k(x-t) h_{1}(t) \mathrm{d} t \mathrm{~d} x \mathrm{~d} y \\
& +\int_{0}^{\infty} \int_{0}^{\infty} y^{n} x^{m} p(x+y) \int_{0}^{x} k(x-t) \mathrm{d} U_{+}^{(2)}(t) \mathrm{d} x \mathrm{~d} y \\
= & : I_{1}+I_{2} .
\end{aligned}
$$

The fact that the integral $I_{2}$ is finite is obvious in view of the claim in (29) and the assumption that $k$ is bounded. For $I_{1}$, let $C_{1}$ be such that $h_{1}(t) \leq C_{1}$ for all $t$. Then, observe that

$$
\int_{0}^{x} k(x-t) h_{1}(t) \mathrm{d} t \leq C_{1}
$$

and, upon using (29) again, this completes the proof.

Remarks. (i) Note that the condition $\int x \mathrm{~d} G(x)<\infty$ in the statement of Proposition 4 is needed to ensure that the ascending ladder height distribution $G_{+}$has a finite first moment (Gut (1987, p. 78)), so that Stone's theorem applies for the associated renewal measure $U_{+}$. 
(ii) It is obvious that by setting $n=0$ and $m=0$ in Proposition 4 we obtain finiteness of the moments for the surplus prior to ruin and, respectively, for the deficit at ruin. Note that the conditions for finiteness of moments for the deficit at ruin, $|U(T)|$, in the Sparre Andersen model, have been given in Cheng et al. (2002).

(iii) It is easily seen from the steps of the proof above that Proposition 4 is also valid for noninteger values of $m$ and $n$ provided that $m \geq 1$.

Based on Proposition 4, we now prove the following more general result.

Theorem 3. Under the assumptions of Proposition 4, assume, in addition, that condition (i) is satisfied. Then (28) holds when $f(x, y \mid 0)$ is replaced by $f(x, y \mid u)$ for an arbitrary value of the surplus $u>0$.

Proof. We have

$$
\begin{aligned}
\int_{0}^{\infty} & \int_{0}^{\infty} y^{n} x^{m} f(x, y \mid u) \mathrm{d} x \mathrm{~d} y \\
& =\int_{0}^{u} \int_{0}^{\infty} y^{n} x^{m} f(x, y \mid u) \mathrm{d} y \mathrm{~d} x+\int_{u}^{\infty} \int_{0}^{\infty} y^{n} x^{m} f(x, y \mid u) \mathrm{d} y \mathrm{~d} x \\
& =: I_{3}+I_{4} .
\end{aligned}
$$

Using the same arguments as in the proof of Proposition 4, it is easily checked that the integral $I_{3}$ is finite, so it suffices to consider the integral $I_{4}$. Using (24) we obtain

$$
\begin{aligned}
I_{4}= & \int_{u}^{\infty} \int_{0}^{\infty} y^{n} x^{m} p(x+y)\left(k \star U_{+}\right)(x-u) \mathrm{d} y \mathrm{~d} x \\
& +\int_{u}^{\infty} \int_{0}^{\infty} y^{n} x^{m} \frac{p(x+y)}{\zeta(0)} \int_{0}^{u}\left(k \star U_{+}\right)(x-u+z) q(z) \mathrm{d} z \mathrm{~d} y \mathrm{~d} x .
\end{aligned}
$$

Since $k$ satisfies condition (i), the key renewal theorem applies so that

$$
\lim _{x \rightarrow \infty}\left(k \star U_{+}\right)(x)=\mu_{0}^{-1} .
$$

Furthermore, the function $k \star U_{+}$is bounded on finite intervals, so we conclude that there exists a constant $C_{2}$ such that $\sup _{x}\left(k \star U_{+}\right)(x)=C_{2}$. This shows, by an appeal to (29) again, that both integrals in (30) are finite and this concludes the proof of the theorem.

\section{Concluding remarks}

Remark 1. Integrating (6) with respect to $x$ over $(0, \infty)$, we obtain

$$
f_{1}(0, y)=\int_{0}^{\infty} p(x+y) \int_{[0, x]} k(x-z) \mathrm{d} U_{+}(z) \mathrm{d} x,
$$

where we have put

$$
f_{1}(0, y)=\int_{0}^{\infty} f(x, y \mid 0) \mathrm{d} x
$$

and we note that this is a density for the deficit at ruin, when the initial surplus is 0 , in (5). For the integral on the right, we use the fact that $U_{+}$has a unit mass at 0 and a density $h$ on $(0, \infty)$ 
to deduce that, for $y \geq 0$,

$$
\begin{aligned}
f_{1}(0, y) & =\int_{0}^{\infty} p(x+y) k(x) \mathrm{d} x+\int_{0}^{\infty} p(x+y) \int_{0}^{x} k(x-z) h(z) \mathrm{d} z \mathrm{~d} x \\
& =g(-y)+\int_{0}^{\infty} h(z) \int_{z}^{\infty} p(x+y) k(x-z) \mathrm{d} x \mathrm{~d} z .
\end{aligned}
$$

Making a simple change of variable and using (16), we obtain

$$
f_{1}(0, y)=g(-y)+\int_{0}^{\infty} h(z) g(-z-y) \mathrm{d} z .
$$

But the Wiener-Hopf identity (Asmussen (1987, Section VII.3)) yields

$$
G_{-}(-y)=\int_{[0, \infty)} G(-y-z) \mathrm{d} U_{+}(z) \text { for any } y>0 .
$$

By differentiation, we obtain

$$
g_{-}(-y)=\int_{[0, \infty)} g(-y-z) \mathrm{d} U_{+}(z)=g(-y)+\int_{0}^{\infty} g(-y-z) h(z) \mathrm{d} z .
$$

From this and (31) we deduce that, for all $y>0$, it holds that $f_{1}(0, y)=\check{g}_{-}(y)$, where $\check{g}$ is a density for $\breve{G}$, and we thus recover (5).

Remark 2. Integrating (22) with respect to $x$ and using Fubini's theorem, it can be shown that the following equation holds:

$$
F(x \mid 0)=\int_{0}^{x}[1-P(t)] k(t) \mathrm{d} t+\int_{[0, x]} \int_{0}^{x-z} \frac{1-P(\xi+z)}{1-P(\xi)} f(\xi \mid 0) \mathrm{d} \xi \mathrm{d} G_{+}(z) .
$$

We note that this can also be obtained by conditioning on the value of the first descending ladder epoch of the random walk $\left\{S_{n}: n=1,2, \ldots\right\}$ and then using arguments similar to those used for the Wiener-Hopf factorisation for random walks; see Kennedy (1994) and Appendix A of Asmussen (2000).

Remark 3. Apart from the surplus prior to ruin and the deficit at ruin, another quantity of interest in the current setup is the amount of claim causing ruin. Let $Z$ be this (defective) random variable, which we note was introduced by Dufresne and Gerber (1988) and studied in detail for the classical Poisson model in Dickson (1993); see also Cai (2004) for some recent results in a more general framework. Assuming that the claim sizes are absolutely continuous with a density $p$, and that the initial surplus is $u$, then $Z$ has a density

$$
g_{u}(x)=\int_{0}^{x} f(z, x-z \mid u) \mathrm{d} z \quad \text { for } x>0 ;
$$

see Dufresne and Gerber (1988). Thus, the results of the previous sections can be applied, without much difficulty, to establish analogous results for $Z$.

Remark 4. A more general problem to the one we have considered in the present paper concerns the behaviour (exact and/or asymptotic) of the more general function defined in (1) for arbitrary $\delta>0$. The results of Gerber and Shiu (1997) for the classical risk model refer to this more general function and we aim to address the issue of generalising the Gerber-Shiu results for the Sparre Andersen model $(\delta>0)$ in the future. 


\section{Acknowledgement}

The authors are grateful for the comments and suggestions of an anonymous referee. These have led to a much improved paper.

\section{References}

Asmussen, S. (1987). Applied Probability and Queues. John Wiley, New York.

Asmussen, S. (1992). Phase-type representations in random walk and queueing problems. Ann. Prob. 20, $772-789$.

Asmussen, S. (2000). Ruin Probabilities. World Scientific, Singapore.

Asmussen, S. AND KlüPPelberg, C. (1996). Large deviation results for subexponential tails, with applications to insurance risk. Stoch. Process. Appl. 64, 105-125.

BADESCU, A. et al. (2005a). Risk processes analysed as fluid queues. Scand. Actuarial J. 2005, 127-141.

BADESCU, A. et al. (2005b). The surplus prior to ruin and the deficit at ruin for a correlated risk process. Scand. Actuarial J. 2005, 433-445.

CAI, J. (2004). Ruin probabilities and penalty functions with stochastic rates of interest. Stoch. Process. Appl. 112, 53-78.

Cheng, Y. And TANG. Q. (2003). Moments of the surplus before ruin and the deficit at ruin in the Erlang(2) risk process. N. Amer. Actuarial J. 7, 1-12.

Cheng, Y. B., TANG, Q. H. And YAng, H. L. (2002). Approximations for moments of deficit at ruin with exponential and subexponential claims. Statist. Prob. Lett. 59, 367-378.

Dickson, D. C. M. (1992). On the distribution of the surplus prior to ruin. Insurance Math. Econom. 11, $191-207$.

Dickson, D. C. M. (1993). On the distribution of the claim causing ruin. Insurance Math. Econom. 12, $143-154$.

Dickson, D. C. M. AND Drekic, S. (2004). The joint distribution of the surplus prior to ruin and the deficit at ruin in some Sparre Andersen models. Insurance Math. Econom. 34, 97-107.

Dickson, D. C. M. And Hipp, C. (2000). Ruin problems for phase-type(2) risk processes. Scand. Actuarial J. 2000, $147-167$

Dickson, D. C. M. And Hipp, C. (2001). On the time to ruin for Erlang(2) risk processes. Insurance Math. Econom. 29, 333-344.

Drekic, S., Dickson, D. C. M., Stanford, D. A. and Willmot, G. E. (2004). On the distribution of the deficit at ruin when claims are phase-type. Scand. Actuarial J. 2004, 105-120.

Dufresne, F. AND Gerber, H. U. (1988). The surpluses immediately before and at ruin, and the amount of claim causing ruin. Insurance Math. Econom. 7, 193-199.

Embrechts, P. ANd Veraverbecke, N. (1982). Estimates for the probability of ruin with special emphasis on the possibility of large claims. Insurance Math. Econom. 1, 55-72.

Embrechts, P., KlüPpelberg, C. And Mikosh, T. (1997). Modelling Extremal Events for Insurance and Finance. Springer, Berlin.

Feller, W. (1971). An Introduction to Probability Theory and Its Applications, Vol. II, 2nd edn. John Wiley, New York.

Gerber, H. U. AND ShiU, E. (1997). The joint distribution of the time of ruin, the surplus immediately before ruin, and the deficit at ruin. Insurance Math. Econom. 21, 129-137.

Gerber, H. U. And Shiu, E. S. W. (1998). On the time value at ruin. N. Amer. Actuarial J. 2, 48-78.

Gerber, H. U. And Shiu, E. S. W. (2003a). Discussion on: 'Moments of the surplus before ruin and the deficit at ruin in the Erlang(2) risk process'. N. Amer. Actuarial J. 7, 117-119.

Gerber, H. U. ANd Shiu, E. S. W. (2003b). Comment on: 'Moments of the surplus before ruin and the deficit at ruin in the Erlang(2) risk process'. N. Amer. Actuarial J. 7(4), 96-101.

Gerber, H. U. And Shiu, E. S. W. (2005). The time value of ruin in a Sparre Andersen model. N. Amer. Actuarial J. 9, 49-84.

Gerber, H. U., Goovaerts, M. J. And KaAs, R. (1987). On the probability and severity of ruin. Astin Bull. 17, $151-163$.

Gut, A. (1987). Stopped Random Walks: Limit Theorems and Applications. Springer, Berlin.

Kennedy, J. (1994). Understanding the Wiener-Hopf factorisation for the simple random walk. J. Appl. Prob. 31, $561-563$.

KLÜPPElBERG, C. (1988). Subexponential distributions and integrated tails. J. Appl. Prob. 25, 132-141.

KlüPpelberg, C. (1989). Subexponential distributions and characterizations of related classes. Prob. Theory Relat. Fields 82, 259-269.

LI, S. M. AND GARrido, J. (2004). On ruin for the Erlang(n) risk process. Insurance Math. Econom. 34, 391-408.

LI, S. M. AND GARRIDO, J. (2005). On a general class of renewal risk process: analysis of the Gerber-Shiu function. Adv. Appl. Prob. 37, 836-856.

Lin, X. S. And Willmot, G. E. (2000). The moments of the time of ruin, the surplus before ruin, and the deficit at ruin. Insurance Math. Econom. 27, 19-44. 
Neuts, M. F. (1981). Matrix-Geometric Solutions in Stochastic Models. Dover, New York.

PicArd, P. AND LefÈvre, C. (1998). The moments of ruin time in the classical risk model with discrete claim size distribution. Insurance Math. Econom. 23, 157-172.

PicARd, P. AND Lefèvre, C. (2003). Probability of ruin in a discrete-time ruin model. J. Appl. Prob. 40, 543-556.

Teugels, J. L. (1975). The class of subexponential distributions. Ann. Prob. 3, 1000-1011.

Willmot, G. E. (2002). Compound geometric residual lifetime distributions and the deficit at ruin. Insurance Math. Econom. 30, 421-438. 